\title{
Políticas SI para la participación ciudadana: análisis regional de la TDT y de la e-Administración
}

\author{
IS policies for citizen participation: regional analysis of \\ DTTV and e-Government
}

\author{
Dra. Natalia Papí Gálvez \\ Universidad de Alicante \\ natalia.p@ua.es \\ Dr. Marcial Murciano Martínez \\ Universidad Autónoma de Barcelona. \\ marcial.murciano@uab.cat
}

Recibido: 5 de Diciembre de 2010 Aceptado: 13 de Febrero de 2011

\begin{abstract}
Resumen
El artículo analiza el estado de la TDT y de la e-Administración de la Comunidad Valenciana (CV) como objetivos prioritarios recogidos en los planes de impulso de la sociedad de la información. Los datos se seleccionan tomando como referencia el modelo de implementación del Plan Nacional de transición de la TDT y de la Unión Europea en materia de e-Administración. Se acude a fuentes secundarias oficiales: los infobarómetros de la Comunidad Valenciana, las encuestas relativas a la sociedad de la información proporcionadas por el INE, los datos proporcionados por la Unión Europea y estudios avalados por estas instituciones. Los resultados apuntan a que el esfuerzo político (y económico) de la CV orientado a la TDT no son claramente distintos a los nacionales. Igualmente, la gran apuesta por la e-Administración no ubica a la CV en una posición líder ante otras comunidades, España o los datos de la TDT.
\end{abstract}

\begin{abstract}
The article analyzes the state of DTTV and e-Government in the Valencian Community (VC) as priority objectives identified in the government plans of information society. The data are selected by reference to the implementation model for the transitional National Plan of DTTV and the European Union in e-Government. It goes to official secondary sources: the infobarometers of Valencia, surveys on the Information Society provided by the INE, data collected in Europe Union i reports of these organizations. The outcomes suggest that the political (and economic) effort in DTTV of VC are not significantly different nationals data. Similarly, the big bet by the e-Government does not put a VC in the leader position to other communities, Spain or DTTV data.
\end{abstract}


Palabras Clave: políticas de comunicación, TDT, e-Administración, sociedad de la información.

Key Words: communication policies, DTTV, e-Government, information society

Sumario: 1. Introducción. 1.1. La Sociedad de la Información como objetivo. 1.2. Hacia la normalización de la TDT. 1.3. La e-Administración como un derecho del ciudadano. 1.4. La apuesta de la Comunidad Valenciana. 2. Método. 3. Resultados. 3.1. Penetración y uso de la TDT en España y en la CV. 3.2. Desarrollo e Implantación de la e-Administración. 3.3. Permanencia o durabilidad: evolución. 3.4. Impacto de las intervenciones públicas. 4. Discusión y conclusiones. 5. Referencias bibliográficas.

Sumary: 1. Introduction. 1.1. The Information Society as an objective. 1.2. Towards the normalisation of DTTV. 1.3. E-government as a citizen's right. 1.4. Commitment to DTTV in the Region of Valencia. 2. Methods. 3. Results. 3.1. DTTV penetration and use in Spain and the Region of Valencia. 3.2. E-government development and implementation. 3.3.

Permanence or durability: evolution. 3.4. Impact of public interventions. 4. Findings and conclusion. 5. Notes and references.

\section{Introducción}

\subsection{La Sociedad de la Información como objetivo}

El artículo muestra el estado de la implantación de la TDT y el desarrollo de la eAdministración de la Comunidad Valenciana (CV) como programas clave para el fomento de la Sociedad de la Información (SI) que pueden y deben contribuir a una mayor participación ciudadana. Ambos proyectos quedan amparados por el contexto internacional y nacional.

El Consejo Europeo extraordinario de Lisboa celebrado en 2000 apuesta por el desarrollo tecnológico como una estrategia de mejora de la competitividad europea entre las economías mundiales. Se establece un reto tecnológico a diez años, y se definen las grandes orientaciones políticas, entre las que se recogen la dotación de infraestructuras y los servicios públicos. Durante estos años, Europa realiza un gran esfuerzo por establecer un marco regulador para tales requerimientos.

Desde la celebración de la Cumbre, España pone en marcha tres programas relacionados con el desarrollo de las nuevas tecnologías: Info XXI, e-España y Plan Avanza. El Plan Avanza está formado por varias fases: pre-avanza, Avanza1 y Avanza2. Las iniciativas de Avanza2 se agrupan en cinco ejes de actuación entre los que se encuentran, en consonancia con el documento europeo i2010, tanto las infraestructuras como los servicios públicos digitales. 
En este marco se reconoce que la integración regional es un factor clave (Cities \& Regions World, 2005). Avanza2 destaca el necesario apoyo de las entidades locales para mejorar la calidad de los servicios prestados por las Administraciones Públicas en Red y para la cual destina, en conjunto, 186 millones de euros sólo en el año 2009. El ámbito local es prioritario también en la dotación de infraestructuras, entre las que destaca la implantación y normalización de la TDT.

\subsection{Hacia la normalización de la TDT}

La TDT representa el giro definitivo hacia la televisión digital y también la "reinvención" de la televisión, en estructura y contenidos, que abre la puerta a la convergencia de los medios.

Plan Nacional de transición de la TDT establece un marco básico de actuación para un cese ordenado y coordinado de las emisiones de televisión con tecnología analógica que garantice el tránsito pleno a la TDT antes del 3 de abril de 2010 (Ministerio de Industria, turismo y comercio, 2007). Genera 90 proyectos de transición repartidos en las diferentes comunidades autónomas y establece 73 áreas técnicas desde el punto de vista radioeléctrico que divide el territorio en ámbitos más pequeños definido por un centro principal de difusión. La CV dispone de cinco de esos proyectos de transición.

El 30 de marzo de 2010 tuvo lugar el acto de bienvenida oficial a la TDT. El Plan nacional se propuso que en abril de ese año llegara al 98\% de la población, aunque la fecha varía según el proyecto de transición. Para la CV, la TDT tenía que estar implantada en aproximadamente la mitad de los municipios de Castellón y la mitad de Valencia, así como tres municipios de Alicante el 31 de diciembre de 2009. Algunos pocos municipios de Valencia han tenido que cumplir con este objetivo antes del 30 de junio. Para el resto, el apagón analógico estaba previsto el mes de abril de este año, 2010.

Recientemente se ha aprobado el Real Decreto 365/2010 por el que se regula la asignación de los múltiples de la TDT tras el cese de las emisiones analógicas, y entrará en vigor la Ley 7/2010 de 31 de marzo General de la Comunicación Audiovisual que afectará tanto al mercado como a los contenidos de la Televisión Digital Terrestre.

En este sentido, la estrategia política de la TDT ha sido cuestionada desde diferentes perspectivas, bien porque las maniobras de última hora puedan derivar en una mayor concentración del mercado (Zallo, 2010), crítica que se hace extensiva al caso valenciano (Corominas, Bonet, Fernández, Guimerá, Sanmartín y Blasco, 2007), con la correspondiente pérdida de las oportunidades para diversificar la oferta y pluralizar los contenidos que ofrecía la TDT; bien por estar aún pendiente la interactividad a través de los servicios y contenidos ofrecidos (Franquet, Ribes, Fernández y Soto, 2009; Franquet; Ribes, Soto y Fernández, 2008). Y con ella se reactiva el debate sobre sus posibilidades comunicativas con el usuario y su contribución al sistema democrático (i.e. Franquet, Ribes, Soto y Fernández, 2009; 
Bustamante, 2008; Prado, Franquet, Soto, Ribes, Fernández, 2008; Prado y Fernández, 2006).

Por lo que no sólo es necesario observar la cobertura de la misma, es decir, si la sociedad tiene garantizado el servicio, también, y sobre todo, es interesante conocer el acceso y la aceptación por parte del usuario o la audiencia de la TDT. De esta forma se puede tener una visión más completa sobre el impacto de esta línea prioritaria de actuación política en el proyecto de la SI desde un enfoque de contribución a la construcción del espacio público.

\subsection{La e-Administración como un derecho del ciudadano}

La e-Administración es un sistema que optimiza recursos, agiliza los procesos y pone en disposición la información al ciudadano y al empresario de forma rápida y eficaz. Por tanto, el uso que los ciudadanos y los empresarios hagan de ella estará en relación directa con la disponibilidad y la calidad del servicio ofrecido por la Administración.

La aprobación de la Ley 11/2007 de Acceso Electrónico de los Ciudadanos a los Servicios Públicos proporciona un gran salto cualitativo en materia de la eficacia de las actuaciones previstas en los planes de impulso de la SI a este respecto. Antes de la 11/2007 se realizaron algunas modificaciones en otras leyes para permitir el establecimiento de registros y notificaciones por medios telemáticos. Sin embargo eran las propias Administraciones las que decidían establecer o no los instrumentos necesarios para que los ciudadanos pudieran comunicarse con ellas a través de la red.

Ahora la Administración pública, sea nacional, regional o local debe transformarse en electrónica para satisfacer el derecho del ciudadano a comunicarse con ella por medios electrónicos. El ciudadano tiene derecho a "obtener información, realizar consultas y alegaciones, formular solicitudes, manifestar consentimiento, entablar pretensiones, efectuar pagos, realizar transacciones y oponerse a las resoluciones y actos administrativos" (España. Jefatura de Estado. Ley 11/2007. art. 6).

Los derechos enumerados por la Ley suponen la puesta en marcha de los mecanismos necesarios por parte de las Administraciones para satisfacerlos. Establece el 31 de diciembre de 2009 como fecha límite para completar este proceso, aunque en el caso de las administraciones regionales y locales esta fecha es más una referencia que una obligación ya que queda sujeta a sus "disponibilidades presupuestarias" (Ley 11/2007. Disposición final tercera).

Esta Ley materializa lo que ya algunos investigadores del derecho administrativo estaban defendiendo (i.e. Tintó i Gimbernat, 2002; Barnés, 2001) o era el aspecto clave de la eadministración (Iturrioz, 2006; Rodríguez, 2006; Castells y Ollé, 2004; Criado y Ramilo, 2001) es decir, que se convierta en "un poderoso instrumento (...) para el mejor servicio al ciudadano, y no el valor de ser una finalidad en sí misma (...)” (Ramiro Sánchez, 2002: 126). 


\subsection{La apuesta de la Comunidad Valenciana}

El Gobierno Valenciano ha puesto en marcha tres planes para el fomento de la SI: PEMAV (1996-1999); Moderniza (2000-2003) y Avantic (2004-2010). Se trata de una de las primeras comunidades autónomas que implantaron planes específicos con este propósito.

En el primer plan la estrategia política se centra sobre todo en la innovación de la Administración, todavía más como usuaria que como garante de infraestructuras y servicios al ciudadano, pese a que el Plan Director de Telecomunicaciones del Gobierno Valenciano, integrado en el PEMAV, recoge también la función del gobierno valenciano y de la Administración en su conjunto como promotores de la SI.

No obstante, el rol de garante aparece años más tarde, con el segundo plan, en el que se destaca la necesidad de garantizar servicios de información y comunicación, para lo que resulta fundamental fomentar el desarrollo de las infraestructuras de telecomunicación necesarias. La puesta en marcha de programas que aborden la cobertura de las telecomunicaciones y la calidad de las infraestructuras se hace evidente con el último plan, Avantic.

Avantic está estructurado en tres macroproyectos: PEVTA (infraestructuras), PETIC (usuarios) y un tercero formado por una serie de programas horizontales. PEVTA es la subdivisión en la que se recoge la implantación de la TDT y la mayor parte de las actuaciones relacionadas con la e-Administración. Esta subdivisión queda formada por un total de tres programas. El segundo programa incorpora la TDT, se trata de su tercera línea de actuación. También en este segundo programa se incluyen proyectos para el desarrollo de la e-Administración que conforman la segunda línea del mismo. Además hay proyectos de e-Administración en la segunda y tercera línea del tercer programa de PEVTA, y también en PETIC.

Avantic es el plan que dispone de más presupuesto. El presupuesto destinado a sus 63 proyectos iniciales alcanza la suma de 2.741 millones de euros, mientras que PEMAV disponía de 9.500 - 10.981 millones de pesetas y MODERNIZA de 38.400 millones de pesetas iniciales - 120 millones de euros finales.

En concreto, la subdivisión PEVTA de Avantic tiene 19 proyectos a los que se destina el $41 \%$ del presupuesto total de Avantic. De esta forma, se hace patente el carácter prioritario de las actuaciones en torno a la implantación de la TDT, ante el apagón analógico del 2010. La media de presupuesto destinado a cada proyecto asciende a 329 millones de euros, pero también para los proyectos relacionados con la e-Administración a lo que hay que sumar el presupuesto destinado en PETIC. De hecho, de los tres grupos en los que se divide PETIC, el mayor esfuerzo presupuestario en su conjunto sigue aplicándose a los programas relacionados con la innovación de la Administración, a los que se destina el $46 \%$ del total del 
programa (PETIC). Por lo que los programas relacionados con la e-Administración, en total, le han adjudicado más financiación que el del fomento de la TDT.

En consecuencia, la implantación de la TDT está presente en la agenda política valenciana, fundamentalmente, en el último plan, y sobre todo, al amparo del impulso nacional e internacional. En cambio, la e-Administración se puede considerar la apuesta más fuerte de la $\mathrm{CV}$, presente desde el primer plan y con una dotación presupuestaria sobresaliente en el último, por lo que cabe esperar resultados incluso más satisfactorios en esta línea de actuación, muy por encima de la media española y, desde luego, encabezando el listado entre las comunidades autónomas restantes. Esta suposición constituye la hipótesis principal de este estudio.

\section{Metodología}

El objetivo de este estudio es describir el estado de la TDT y de la e-Administración de la CV a través de una selección justificada de indicadores de entre los de seguimiento e impacto considerados por los organismos oficiales. Los datos se eligen tomando como referencia el modelo de implementación del Plan Nacional de transición de la TDT y de la Unión Europea en materia de e-Administración.

El Plan Nacional de transición de la TDT propone varios indicadores que proporcionan información suficiente del avance el proyecto en sus diferentes etapas: venta de receptores, actualización de antenas, cobertura, intención y satisfacción ciudadana, equipamientos red de T \& D, audiencias TDT y utilización de servicios.

Por las implicaciones que la TDT tiene en la comunicación mediática y debido a las fechas de los últimos datos disponibles proporcionados por las fuentes secundarias oficiales, en las que la transición a la TDT debería estar casi completada, son especialmente relevantes los indicadores que aportan información relativa a los hogares y a los usuarios. En consecuencia, se hace especial hincapié en la penetración de la TDT en los hogares ya que este dato engloba la cobertura de la TDT (el objetivo mínimo establecido) y, además, proporciona una idea del acceso y uso de la población hacia la misma.

En la medición de la e-Administración se definen varias fases de desarrollo. Según el estudio de Capgemini Consulting (2010), se puede dividir en cinco etapas (Previa, Información, Interacción unidireccional, Interacción bidireccional y Transacción o interacción totalmente electrónica), debe ofrecer 26 servicios y debe dirigirse a dos tipos de públicos: ciudadanos y empresas. Este modelo se basa en la Ley 11/2007 y queda avalado por la Unión Europea. En consecuencia, en este estudio se observan los servicios disponibles para el ciudadano y el empresario a través de Internet. Igualmente, en la medida que la disponibilidad de datos lo permite, se observan los usos de la e-Administración por parte de los ciudadanos. 
Finalmente, los ejes estratégicos que marcan tanto la agenda nacional como regional, y de acuerdo con la Cumbre de Lisboa, tenían el último propósito de hacer crecer el sector de las nuevas tecnologías. En consecuencia, también se seleccionan el PIB/cápita y el volumen de negocio en SI como los indicadores más representativos del impacto socioeconómico esperado. Se observará también los hogares con banda ancha como aproximación al equipamiento y, por tanto, a las posibilidades de acceso a la e-Administración de los ciudadanos y su consumo de otros servicios de la SI.

Sería deseable observar la creación de empleo vinculado a sectores relacionados con las nuevas tecnologías pero la disponibilidad de datos es limitada. Sólo se conoce el número de ocupados desde el 2003 al 2007 a nivel nacional por lo que no es posible hacer una comparativa con la Comunidad Valenciana.

Los indicadores se seleccionan de las encuestas oficiales y de los estudios disponibles realizados al amparo de la Unión Europea. Así, se consultan los Infobarómetros proporcionados por la Generalitat Valenciana, las encuestas realizadas por el Instituto Nacional de Estadística y los estudios facilitados por el Ministerio de la Presidencia.

Todos los datos seleccionados proporcionan información, en última instancia, de los resultados de las políticas de comunicación recogidas en los planes (Alvira, 2002). Por tanto, a través de los mismos se puede realizar una valoración de las actuaciones en su conjunto; del esfuerzo realizado por la CV para cumplir con los objetivos marcados en materia de TDT y e-Administración.

Para ello, se seguirá una doble estrategia: por un lado, la comparación tendrá lugar entre las últimas cifras disponibles de la CV y las nacionales (perspectiva transversal) y, por otro lado, se observarán los datos de la CV en varios años (perspectiva longitudinal). En concreto, se tratará de observar la evolución de los datos desde que entra en vigor el último plan de la CV, es decir, desde el año 2004, aunque no siempre es posible, ya que algunos de los indicadores más orientados al usuario aparecen a partir de 2006, cuando entra en vigor el plan Avanza.

Los resultados se estructuran en torno a estos análisis y a la disponibilidad de los mismos en las fuentes secundarias indicadas. Así, en primer lugar, se exponen los datos de los indicadores seleccionados para la TDT (mes de marzo de 2010) y para la e-Administración (año 2009-2010) tanto a nivel nacional como para la CV y se comparan con las cifras proporcionadas hace uno y dos años, incluso con las observadas para otras comunidades autónomas. A continuación se recogen los datos por años para obtener una visión más completa de la permanencia o durabilidad de los posibles efectos. Se muestran los datos de penetración de la TDT desde 2004 hasta 2009-10 nacionales y de la CV.

Sobre la disponibilidad de servicios de la e-Administración, a nivel nacional, se tienen cifras de cuatro años (2006-2007, 2007-2008, 2008-2009 y 2009-2010). Se utilizarán también los 
infobarómetros de la CV porque proporcionan otro tipo de información, complementaria al resto. Con respecto a la e-Administración, los informes valencianos se centran en las corporaciones locales (y no en la Administración regional). En concreto los datos se basan en 232 Ayuntamientos de los 542 posibles.

En relación con los indicadores de impacto socioeconómico, las fuentes secundarias permiten su análisis en un periodo más amplio por lo que se recogen datos desde 2004, a nivel nacional y para la CV.

\section{Resultados}

\subsection{Penetración y uso de la TDT en España y en la CV}

En términos globales, la cobertura de la TDT en España en marzo de 2010 supera, como cabe esperar, el 98\%. Cuando se trata de la penetración, la cifra es inferior (89\%). Aunque, en comparación, la penetración de la TDT en España es más elevada a la que proporcionan otros países europeos como es Reino Unido, Francia o Italia. Esta alta penetración genera una cuota de pantalla del $68 \%$ en marzo de 2010. En un año el incremento de la penetración y de la cuota ha sido de casi el doble.

El cálculo de cuota de pantalla proporciona una idea del uso que la población realiza de la televisión, en este caso, según el medio de distribución, en tanto audiencia de TDT sobre la audiencia de la televisión. El hecho de que el porcentaje de penetración y el de la cuota no coincidan se debe a que existen emisiones analógicas. La cuota de la analógica asciende al $12 \%$ en el mes de marzo. Y a pesar del encendido digital definitivo en el mes de abril, con el que se observará un evidente descenso de la cuota por televisión analógica terrestre, se prevé un porcentaje residual de analógica a través, fundamentalmente, del satélite.

El análisis por comunidades autónomas proporciona datos por encima del $73 \%$ de penetración, en el mismo mes de referencia. La CV ocupa el noveno lugar en este indicador con un $88 \%$. El análisis de la cuota de pantalla por comunidades autónomas muestra mayores diferencias entre ellas: desde el $43 \%$ del País Vasco hasta el $76 \%$ de Cataluña en marzo de 2010. La CV tiene un 69\% de cuota, dato algo superior al porcentaje nacional, lo que le sitúa en el sexto lugar de entre las comunidades autónomas. En un año, el crecimiento del número de hogares con TDT y de la cuota de pantalla ha sido destacado en todos los casos, con fuertes incrementos en las últimas semanas.

Los datos de penetración de la TDT de los Infobarómetros son mayores que las cifras nacionales oficiales pues incluyen toda la televisión digital, también la de pago por cable. No obstante, si sólo se cuenta con la TDT (gratuita), la penetración desciende de 64,5\% al 49\% (en 2008, últimos datos disponibles). Con todo, algo más del $24 \%$ de hogares sin televisión digital en 2008 dicen tener intención de incorporar la TDT gratuita, cuando este porcentaje no subía del 7\% en el año 2007. Por ello se puede vaticinar una penetración mayor en 2009. 
Esto es así más si se tiene en cuenta la valoración que recibe la televisión digital en 2008, ya que los valencianos aceptan este tipo de televisión situándose en un 7 sobre una escala de rechazo/aceptación, en la que el 10 significa máxima aceptación.

\subsection{Desarrollo e Implantación de la e-Administración}

Según el informe europeo i2010 (EC, 2009) también España obtiene buenos resultados en el desarrollo e implantación de la e-Administración cuando se trata de la disponibilidad de los servicios básicos públicos a través de la red. Se ubica en el noveno lugar. Sin embargo, cuando se trata del uso que los ciudadanos y las empresas realizan de la e-Administración, España se sitúa entre el puesto 13 y 25, según indicador (2008). Además, la normalización del uso de las TIC ha sido más lenta en España que en otros países, al menos, desde el año 2004, pese a que ya en 2001 se pone en marcha el primer plan (Info XXI) y en 2006 entra en vigor el Plan Avanza.

Entre comunidades autónomas (Capgemini Consulting, 2010) la CV estaría cumpliendo con el $76 \%$ en términos de disponibilidad de los 26 servicios, y con un porcentaje muy similar entre los destinados a los ciudadanos y los destinados a empresas. Esta Comunidad está levemente por debajo de la media nacional (77\%). Los datos sitúa a la CV en la novena posición del ranking en el estudio de 2010.

En contraste con la TDT, el crecimiento en un año no es espectacular. En el estudio de 2008, la CV obtenía un índice de 69\%, y en 2009 del 71\%, en noveno y décimo lugar del ranking según año. Estos datos suponen que de 26 servicios posibles en la CV (16 dirigidos al ciudadano y 10 al empresario) sólo uno más ha podido alcanzar el estadio 4 en un año. Tampoco la CV lidera el ranking cuando se observa la disponibilidad de los servicios por tipo de público.

El uso que hace la población de Internet para comunicarse con la Administración se centra fundamentalmente en la mera obtención de información (figura 1). Según los datos de 2010, el $57 \%$ de la población española de entre 16 y 74 años ha utilizado este medio electrónico con este objetivo, el $37 \%$ además se ha descargado algún documento y el $25 \%$ ha rellenado algún formulario. Por tanto, sólo el $25 \%$ ha completado todo el proceso.

Los porcentajes para la CV no se alejan de los mostrados para España en los dos primeros casos (58\%, 37\%) y están por encima de la media española en el tercero (28\%) (figura 1$)$. Se aprecia un aumento de población que se comunica con la Administración en este periodo (datos 2009 nacional: 57, 35, 20; datos 2009 CV: 54, 26, 22; y datos 2008 nacional: 55, 35, 22; datos 2008 CV: 53, 34 y 19). Según los datos, la población valenciana que completa todo el proceso crece en mayor medida que la población española tomada en su conjunto.

Sin embargo, entre las diecinueve comunidades autónomas, los datos de la encuesta de 2010 sitúan a la CV en el sexto lugar mientras que en el año 2009 estaba en el cuarto 
(población que rellena formularios). A esta posición se le debe unir la evidente observación que no toda la población valenciana que establece algún nivel de comunicación con la Administración pública lo hace con algún organismo de su Comunidad ${ }^{1}$.

Figura I. Porcentaje de población de 16 a 74 años que establece comunicación por Internet con las Administraciones Públicas en los últimos 12 meses por CC.AA y tipo de servicio. Ranking de CC.AA por población que completa todo el proceso (col. 3).

\begin{tabular}{|l|r|r|r|}
\hline & $\begin{array}{r}\text { 1. Obtener } \\
\text { información }\end{array}$ & $\begin{array}{l}\text { 2. Descargar } \\
\text { documentos }\end{array}$ & $\begin{array}{l}\text { 3. Rellenar } \\
\text { formularios }\end{array}$ \\
\hline Galicia & 63,8 & 43,5 & 31,7 \\
\hline Ceuta & 57,6 & 52,3 & 31 \\
\hline Murcia (Región de) & 58,6 & 41,9 & 30,1 \\
\hline Asturias (Principado de) & 61,6 & 38,8 & 29 \\
\hline Castilla y León & 66 & 40,2 & 28,2 \\
\hline Comunitat Valenciana & 58,4 & 36,6 & 27,8 \\
\hline Extremadura & 54,7 & 39,7 & 27,5 \\
\hline Aragón & 62,5 & 40,6 & 26,8 \\
\hline Total nacional & 57 & 36,5 & 24,9 \\
\hline Madrid (Comunidad de) & 65,5 & 43,8 & 24,5 \\
\hline Andalucía & 50,9 & 33,7 & 24,2 \\
\hline Cataluña & 54,1 & 31,8 & 24 \\
\hline Castilla-La Mancha & 58,1 & 41,8 & 23,5 \\
\hline Melilla & 39,3 & 26 & 22,1 \\
\hline País Vasco & 53,7 & 31,2 & 21,4 \\
\hline Cantabria & 52 & 34,3 & 20 \\
\hline Canarias & 47,4 & 28,1 & 19,7 \\
\hline Rioja (La) & 58,3 & 34,3 & 19,5 \\
\hline Navarra (Comunidad Foral de) & 56 & 31,9 & 18,3 \\
\hline Balears (Illes) & 44,2 & 26,8 & 18,2 \\
\hline
\end{tabular}

Fuente: INE, Encuesta sobre equipamiento y uso de tecnologías de la información y de la comunicación en los hogares, 2010. Nota: se utilizan los datos de los últimos 12 meses y no de los 3 meses porque pueden verse menos afectados por los diferentes periodos de trámites y pagos establecidos entre Administraciones.

\subsection{Permanencia o durabilidad: evolución}

Los datos de penetración de la TDT anual en los hogares que ofrece el INE muestran la siguiente evolución: 86\% (2010), 56,8\% (2009), 37,4\% (2008), 22,8\% (2007), 19,4\% (2은 semestre 2006), 12,1\% (1 semestre 2006), 3,30\% (2 semestre 2005) y 2,37\% (1\% semestre 2005), 1,95\% (2004). Lógicamente en $2010-2011$ se esperan datos cercanos al $100 \%$.

${ }^{1}$ En los últimos tres meses de la encuesta de 2009, sólo el 24,7\% de los valencianos buscaron información en Administraciones de su Comunidad.

Facultad de Ciencias de la Información - Universidad de La Laguna Avenida César Manrique, s/n; Campus de Guajara 38071 La Laguna, Tenerife (Islas Canarias - España) 
En la CV, el $84 \%$ de los hogares tienen este tipo de televisión según los últimos datos (2010), 63,5\% (2009), 43\% (2008). En 2007 sólo el 26,1\%. En 2006 los datos son 20,9 y 10,2\%, para el segundo y primer semestre respectivamente, y de 2,45 y 1,47 en el primer y segundo semestre del año 2005. En 2004 era sólo del 0,78\%.

El aumento es notable a nivel nacional pero ha sido espectacular en la CV. De nuevo, según los infobarómetros se puede advertir un gran salto en los dos últimos años que ayuda a observar el esfuerzo realizado en la implantación de este tipo de televisión en la CV².

En cambio, la disponibilidad de servicios públicos (e-Administración) para la CV no sufren estas variaciones entre los años con datos (2007, 2008, 2009, 2010), aunque se observa una tendencia creciente (figura 2) mayor en el caso de la media española.

Figura II. e-Administración (índice de disponibilidad para todos los servicios) Nacional y CV.

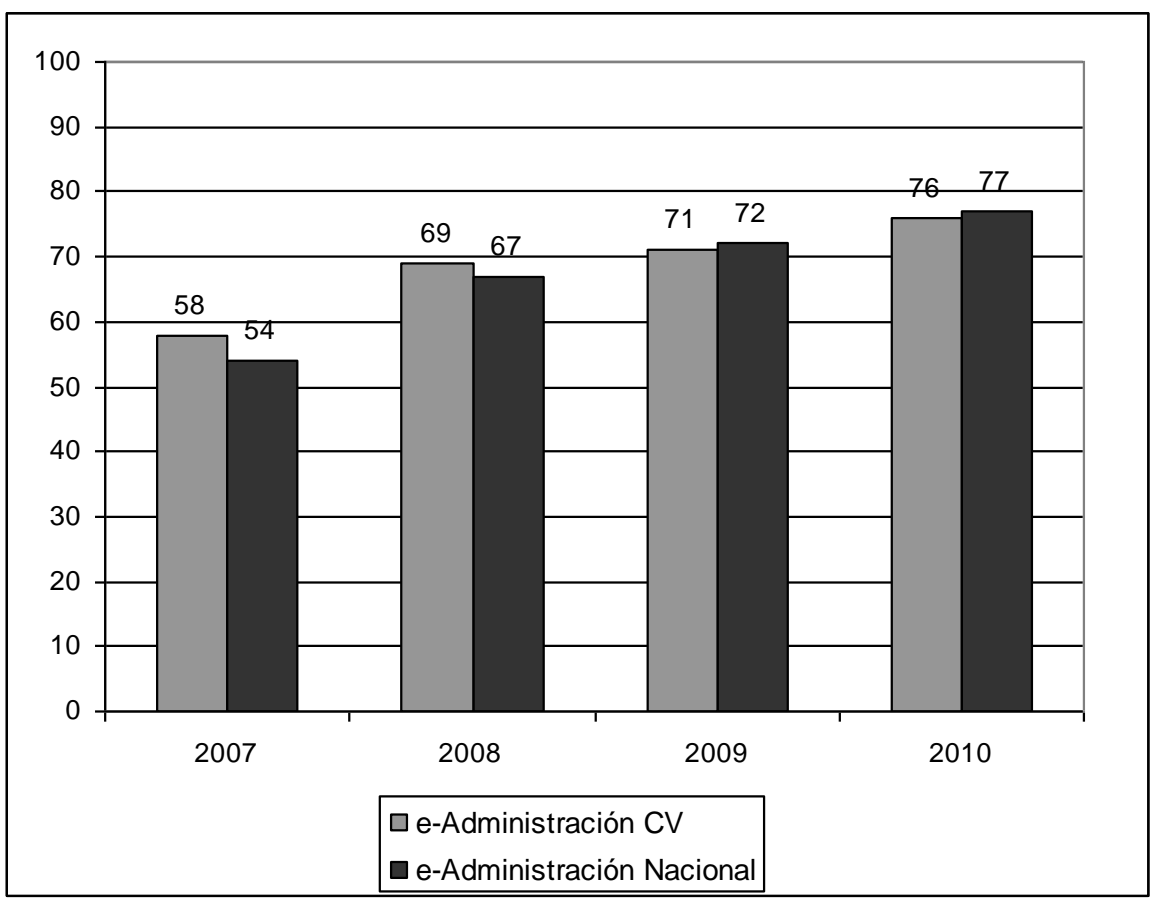

Fuente: Elaboración propia a partir de los datos de Capgemini Consulting

Con respecto a la e-Administración según datos de los Infobarómetros, el 95\% de los Ayuntamientos cuentan con una página web, la gran mayoría con dominio propio (98\%), de

${ }^{2}$ En 2008 la penetración es del 49\% (TDT gratuita), en 2007 el 40,5\% de hogares tenían televisión digital de los cuales el $23,7 \%$ era TDT (gratuita). No se disponen de datos para 2006. En 2005, el 18,9\% de los hogares tienen algún tipo de televisión digital, y en 2004 asciende a 20,5\% hogares. Los datos de los dos primeros años (2004 y 2005) están sobreestimados ya que hacen referencia, fundamentalmente, a la televisión de pago. 
las cuales el $6 \%$ permiten realizar trámites completos on-line. Este porcentaje aumenta hasta el $21 \%$ cuando los ayuntamientos pertenecen a los municipios con mayor población (más de 10.000 habitantes).

En 2007, el 82,3\% tenían una página web oficial, la gran mayoría con dominio propio, de las cuales el $4,2 \%$ permiten realizar trámites on-line completos, el $12 \%$ en el caso de ayuntamientos de municipios de más de 10.000 habitantes.

En 2006 y en 2005 las cifras son las siguientes, respectivamente: 61,9\% y 48,9\% página web oficial (82,5\% y 78,8\% con dominio propio), $15,7 \%$ y $8,8 \%$ trámites completos on-line, $37,5 \%$ y $17,9 \%$ trámites completos on-line por ayuntamientos de más de 10.000 habitantes.

Se observa (figura 3), por tanto, que ha habido un aumento progresivo en cuatro años en la creación de páginas web en las corporaciones locales de la $\mathrm{CV}$, lo que se puede interpretar como un gran salto para el e-Gobierno. En cuanto a los servicios ${ }^{3}$, no existe un incremento constante en los cuatro años. Sólo una pequeña parte de las páginas web de los ayuntamientos disponen del mismo, incluso si se atiende únicamente a los datos de los ayuntamientos que disponen de web de municipios de más de 10.000 habitantes.

Figura III. Ayuntamientos en la CV con página web y páginas web que permiten hacer trámites on-line y que permiten realizar trámites on-line de Ayuntamientos de hábitats superiores a 10.000 habitantes.

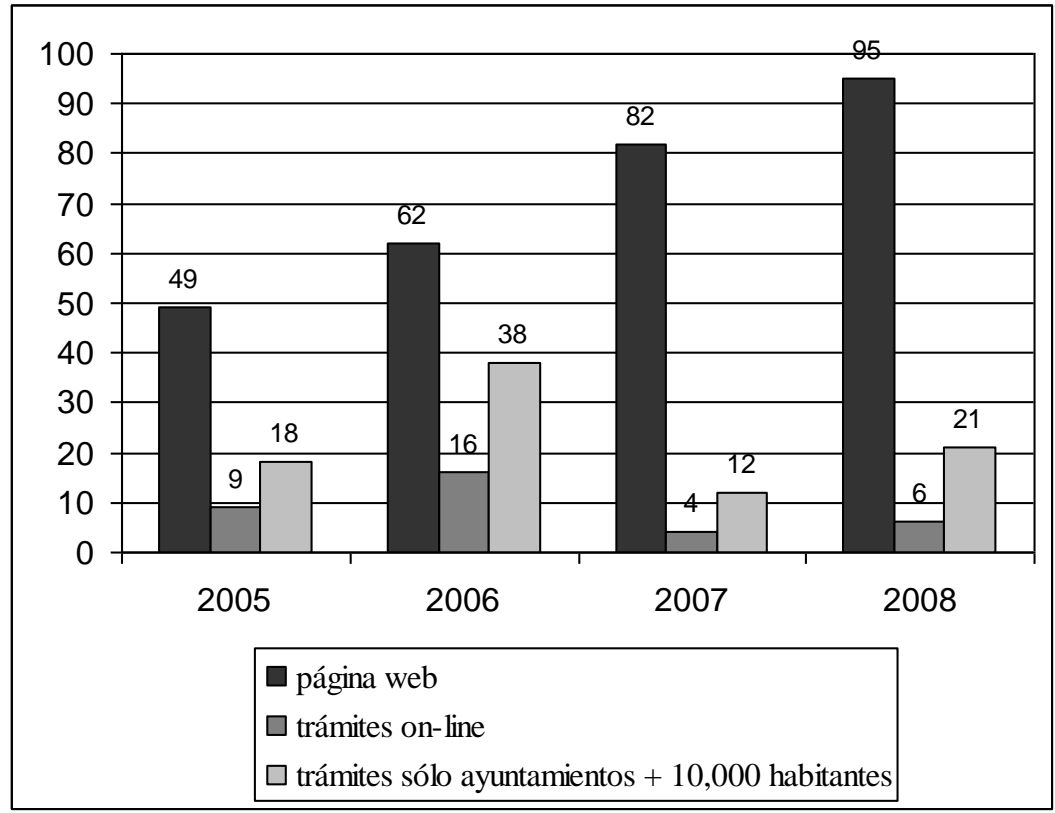

Fuente: Elaboración propia a partir de los datos de los Infobarómetros e-Gobierno de la CV.

\footnotetext{
${ }^{3}$ Se ha seleccionado la posibilidad de realizar trámites on-line por analogía con los criterios nacionales, en los que el último estadio era rellenar un formulario.
}

Facultad de Ciencias de la Información - Universidad de La Laguna

Avenida César Manrique, s/n; Campus de Guajara

38071 La Laguna, Tenerife (Islas Canarias - España) 


\subsection{Impacto de las intervenciones públicas}

EI PIB cápita de la CV se aleja más del de España justamente cuando comienza a desarrollarse las fases intermedias del plan Avanza y se plantea el plan Avantic (figura 4). En el último año se aprecia un descenso del PIB cápita por el efecto de la crisis económica pero, además, aumenta levemente la diferencia entre el PIB cápita de la CV con toda España.

Figura IV. Evolución del PIB cápita nacional y de la CV.

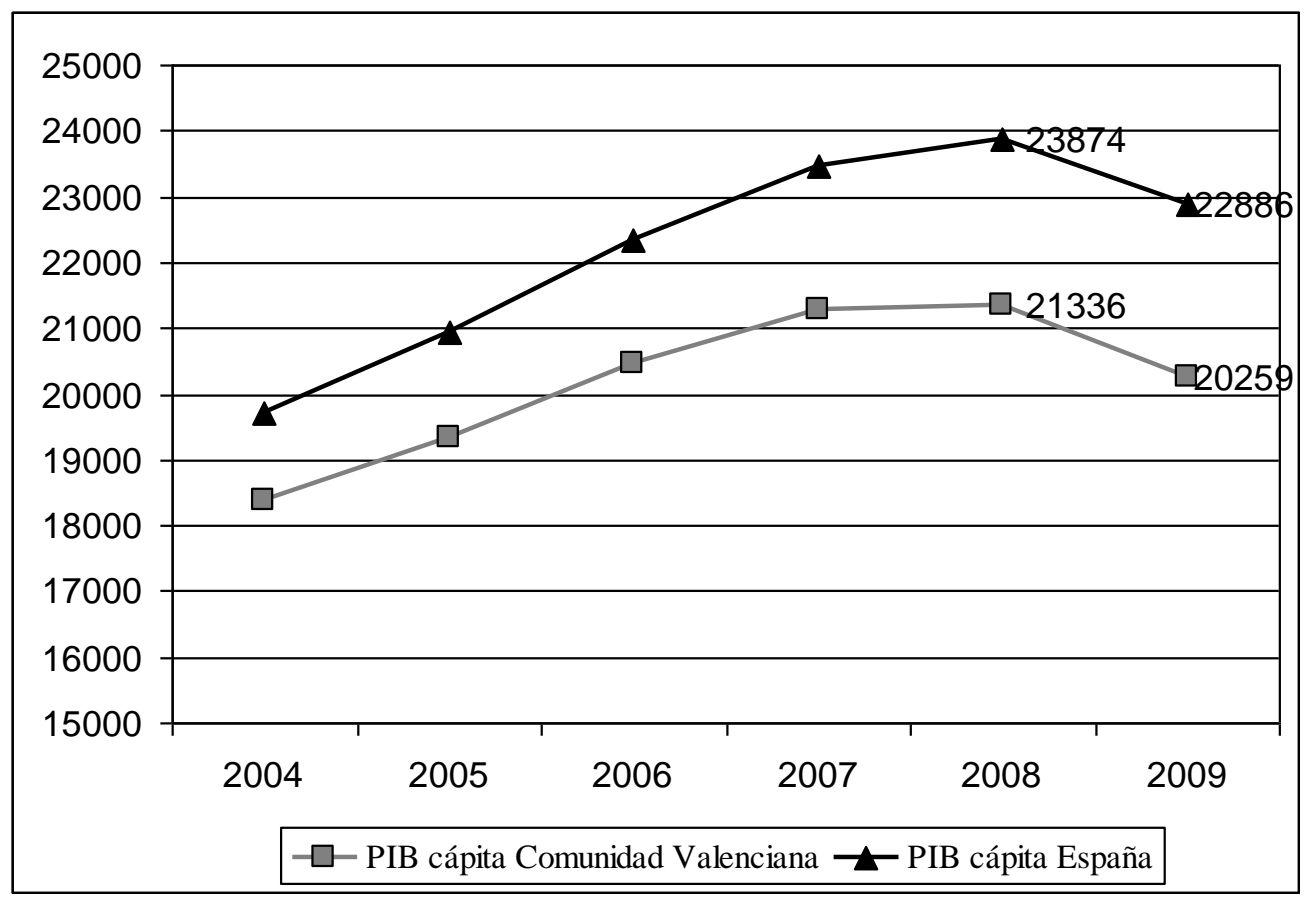

Fuente: Elaboración propia a partir de datos del INE. 2007 y 2008 datos provisionales y 2009 primera estimación.

La diferencia entre la CV y el volumen de negocio para la SI es muy significativa ya que dicho indicador viene a medir una de las directrices marcadas a nivel internacional (figura 5). No obstante, las gráficas no muestran un aumento en este tipo de actividades desde 2004. De hecho, si se toman los dos últimos datos, se advierte que incluso la contribución de este sector al PIB ha descendido. En los dos últimos años el descenso en el caso de la CV en volumen de negocio SI es mayor que en el caso de España. 
Figura V. Volumen de negocio en SI nacional y de la CV sobre PIB nacional y regional.

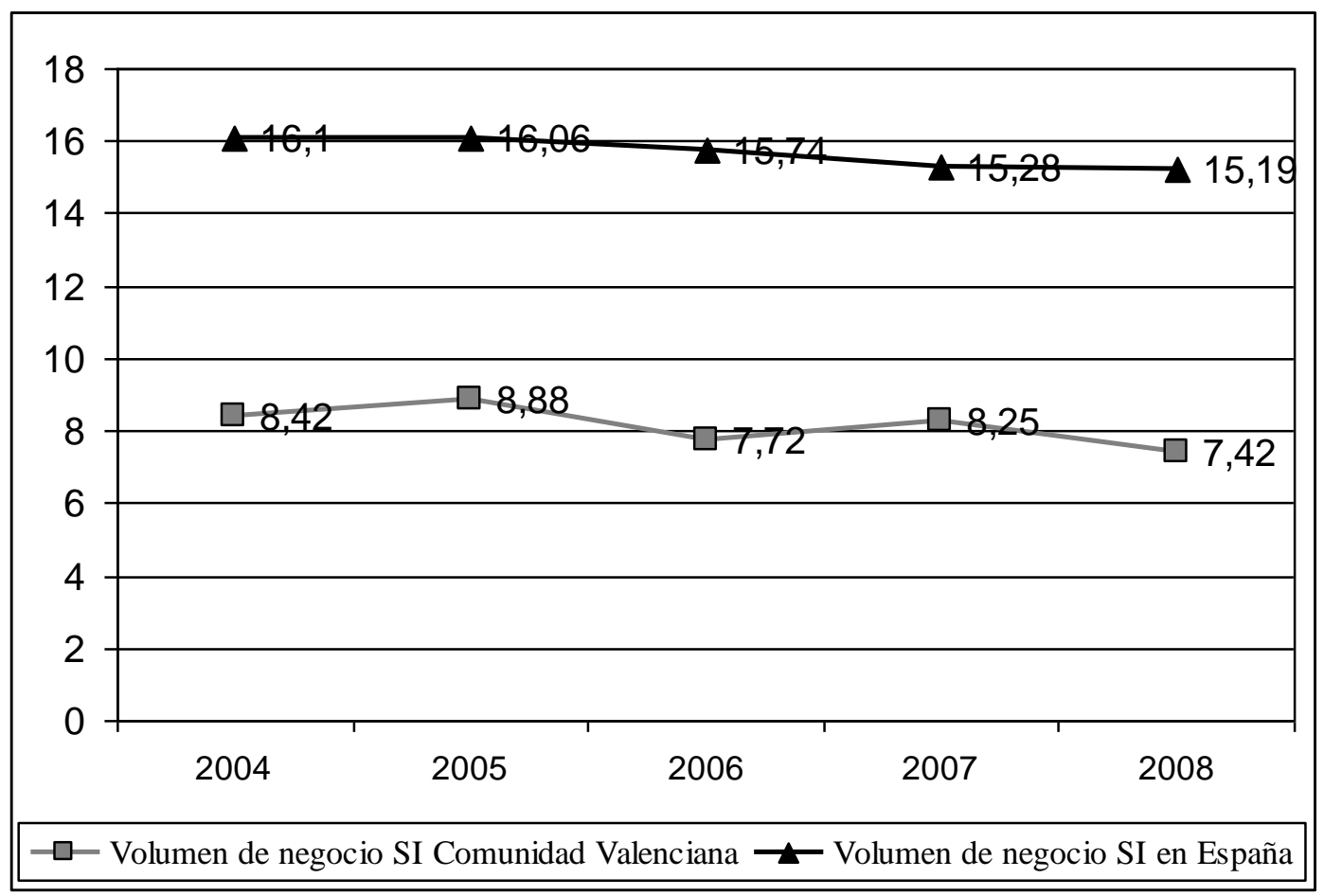

Fuente: Elaboración propia a partir de datos del INE. Dato elaborado con la información proporcionada por la Encuesta Anual de Servicios y las cuentas regionales.

Los hogares con banda ancha (figura 6) proporcionan una idea de la preparación de los hogares para utilizar los servicios que ofrece la SI, como por ejemplo todos los relativos al acceso a los servicios públicos. Al contrario de lo que ocurría con el volumen de negocio, aquí hay un crecimiento constante. Los datos de España son más positivos que los de la CV y en los dos últimos años se aprecia que el incremento de los hogares con banda ancha es mayor en el caso de España que en el caso de la CV. 
Figura VI. Hogares con banda ancha nacional y de la CV.

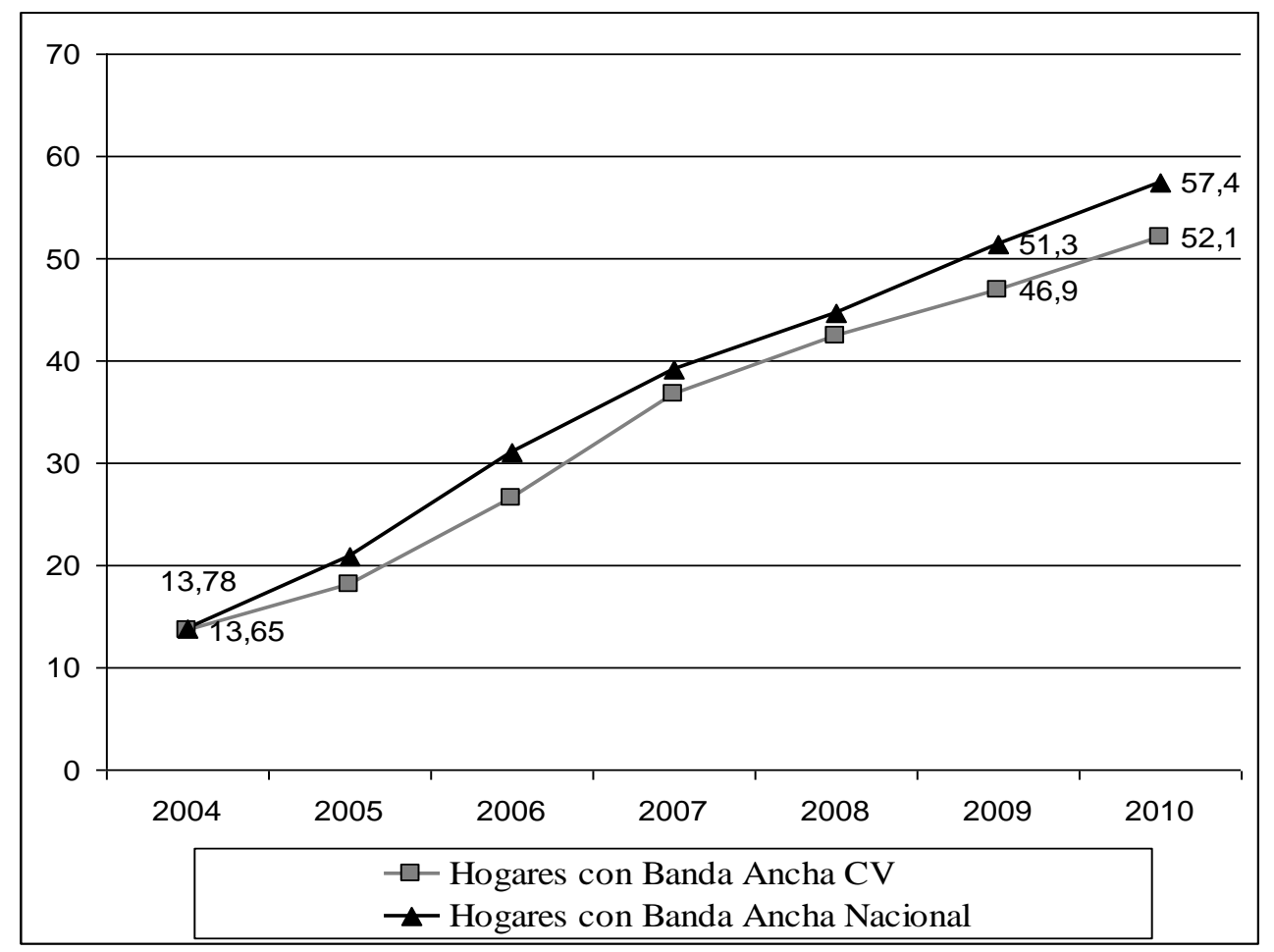

Fuente: Elaboración propia a partir de datos del INE. Encuesta sobre equipamiento y uso de tecnologías de la información y de la comunicación en los hogares, 2010.

\section{Discusión y conclusiones}

Los ejes estratégicos que marcan tanto la agenda nacional como regional, y de acuerdo con la Cumbre de Lisboa, tenían el último propósito de hacer crecer el sector de las nuevas tecnologías y normalizar su uso. En consecuencia, se debe observar el comportamiento tanto de indicadores relacionados con el crecimiento económico como con la penetración y utilización de las TICs por parte de la población. En esta ocasión, y en lo que afecta a la normalización, el análisis se centra en la TDT y en la e-Administración.

Se debe considerar que la TDT ha sido fuertemente impulsada en las anteriores semanas del inminente apagón analógico, aunque la alta penetración en los hogares de la TDT puede dar una falsa idea de normalización de este tipo de televisión. La diferencia entre las cifras de penetración y de cuota de pantalla, pese a ser relativamente elevada, lleva a replantear la normalización en el uso, es decir, que quede completamente integrada en la vida de los usuarios. En este análisis la CV no sale mal parada aunque, de entre todas las comunidades, 
tampoco lidera el encendido digital terrestre. La posición de la CV en todos los indicadores revisados es intermedia, muy cercana a la media nacional.

Con los datos actuales, se debe afirmar que el esfuerzo político (y económico) de la CV orientado a programas para la implantación y normalización de la TDT integrados en el último plan (Avantic) no genera resultados claramente distintos a los nacionales. Ciertamente se aprecia un mayor incremento en los últimos años en la CV en relación con España, fundamentalmente a partir del segundo semestre de 2006, cuando entra en vigor el Plan Avanza (nacional), y cuando Avantic (el plan regional) ya llevaba funcionando dos años. A la luz de estos datos, el Plan Avanza parece haber jugado un importante papel en la implantación de la TDT en la CV. Los planes del gobierno valenciano no han proporcionado una ventaja suficiente para que la comunidad valenciana destaque notablemente por encima de la media nacional.

Ahora bien, a favor de la CV, se observa una gran aceptación de la televisión digital por parte de la población que queda confirmada por la intención de obtener la TDT declarada por los valencianos en 2008, todavía a un año y medio del apagón analógico.

En cuanto a la e-Administración, recuérdese que en el plan valenciano (Avantic) había un programa especialmente centrado en la implantación de la TDT y más de tres relacionados con la e-Administración, con su debida dotación presupuestaria. Además, este predominio de la e-Administración con respecto al resto de ejes, queda reflejado en los dos planes regionales anteriores. Por tanto, parecía lógico esperar que los datos de la e-Administración fueran superiores al resto de España, o al menos que la CV se hubiera encontrado entre las primeras comunidades. Sin embargo, estos esfuerzos no se reflejan en una situación que ubique a la CV a la cabeza o entre las primeras comunidades autónomas.

De nuevo, a favor de la población valenciana, hay una integración mayor que en otras comunidades en el uso de las webs para completar todo el proceso de transacción con la Administración. Igualmente, se debe reconocer un esfuerzo por parte de los Ayuntamientos en la generación de webs oficiales, aunque menos de la tercera parte de los que se ubican en municipios con tamaños superiores a 10.000 habitantes ofrezcan la posibilidad de realizar trámites on-line en 2008.

También era plausible la consideración de que los resultados de la e-Administración reflejaran una realidad que ubicara a la CV en una posición mejor que la que obtuviera por la implantación y normalización de la TDT, debido a la relevancia que ésta adquiere desde la concepción del primer Plan. En cambio, los datos apuntan a que aún hay muchos servicios que deben llegar a la fase final. Y esto es así tanto para la media española como para la Comunidad Valenciana. Según estos datos, no se cumpliría el horizonte temporal de la Ley 11/2007, al establecer como fecha límite el 1 de enero de 2010. 
Finalmente, de acuerdo con el propósito de hacer crecer el sector de las nuevas tecnologías de la Cumbre de Lisboa, los indicadores de impacto socioeconómico seleccionados también reflejan posiciones de la CV inferiores a la media nacional. Para encontrar explicaciones se deben considerar otros factores estructurales, tales como la capacidad de crecimiento de la economía de la región. En este sentido, entre las causas que subyacen en los resultados de la Comunidad, por debajo de la media nacional, se debe destacar que la Valenciana tiene, y sigue teniendo, un tejido empresarial de pequeña y mediana empresa, con poca capacidad para desarrollar un volumen de negocio SI basado en innovación, que genere efectos a largo plazo y que tenga mayores posibilidades de crecimiento. Además el sector turismo es fundamental en la economía de muchos municipios y, por tanto, muy vulnerable a las crisis económicas.

No obstante el volumen de negocio en sectores afines a la SI en relación con el PIB no ha crecido en cuatro años ni en España ni en la CV, lo que hace sospechar del posible impacto a nivel macroeconómico tanto de los planes regionales como de los nacionales. Quizás el impacto queda reflejado más en el equipamiento y consumo, por tanto, de los hogares y de los ciudadanos que en el impulso de un sector productivo en el que impere la innovación y la tecnología. En consecuencia, el propósito último de la Cumbre de Lisboa celebrada en el año 2000 se ha cumplido sólo en una pequeña parte, ocho años más tarde.

Actualmente se puede hacer una aproximación al análisis del impacto generado por las intervenciones públicas con los indicadores disponibles. Sin embargo, la gran crisis económica devenida en los últimos años hace pensar que los datos no serán tan positivos como los deseados, entre otras cuestiones porque una buena parte de las expectativas se depositaron en la creación de empleo.

\section{Bibliografía}

Alvira Martín, F. (2002): Metodología de la evaluación de programas, Cuadernos Metodológicos, número 2. Madrid: CIS.

Asociación para la implantación y el desarrollo de la televisión digital terrestre en España (2010): “Informe Mensual, Abril 2010, número 38". Madrid: ImpulsaTDT. Recuperado el 1 de junio de 2010 de: http://www.impulsatdt.es/pdf/boletin/INFORME ABRIL 2010.pdf

--- (2009): “Informe Mensual, Abril 2009, número 27”. Madrid: ImpulsaTDT. Recuperado el 1 de junio de 2010 de: http://www.impulsatdt.es/pdf/boletin/INFORME ABRIL 09.pdf

Barnés, J. (2001): "Una reflexión introductoria sobre el Derecho Administrativo y la Administración Pública de la SI y del Conocimiento", Revista Andaluza de Administración Pública, 40, pp 25-76. 
Bustamante, E. (2008): La televisión digital terrestre en España. Por un sistema televisivo de futuro acorde con una democracia de calidad. Documento de trabajo 129/2008. Madrid: Fundación Alternativas.

Capgemini Consulting (2007): Estudio Comparativo 2007 de los Servicios Públicos on-line en las CC AA. Madrid: Fundación Orange-Capgemini Consulting. Recuperado el 3 de febrero de 2010 de: http://www.fundacionorange.es/areas/25 publicaciones/eadministracion2007.pdf

--- (2008): Estudio Comparativo 2008 de los Servicios Públicos on-line en las CC AA. Madrid: Fundación Orange-Capgemini Consulting. Recuperado el 3 de febrero de 2010 de: http://www.fundacionorange.es/areas/25 publicaciones/eadministracion2008.pdf --- (2009): Estudio Comparativo 2009 de los Servicios Públicos on-line en las CC AA. Madrid: Fundación Orange-Capgemini Consulting. Recuperado el 3 de febrero de 2010 de: http://www.fundacionorange.es/areas/25 publicaciones/eadministracion2009.pdf --- (2010): Estudio Comparativo 2010 de los Servicios Públicos on-line en las CC AA. Madrid: Fundación Orange-Capgemini Consulting. Recuperado el 5 de septiembre de 2010 de: http://www.informeeespana.es/docs/Estudio Comparativo 2010 Servicios on line.pdf

Castells, M. y Ollé, E. (2004): El modelo Barcelona II: el Ayuntamiento de Barcelona en la sociedad red de Catalunya, Informe de investigación. Barcelona: UOC. Recuperado el 3 de febrero de 2010 de: http://www.uoc.edu/in3/pic/esp/pdf/PIC Ajuntament esp.pdf

CEVALSI (2004): Infobarómetro social de la Comunidad Valenciana 2004. Valencia: Fundación Oficina Valenciana para la SI.

--- (2006): Infobarómetro social de la Comunidad Valenciana 2005, Resumen ejecutivo. Valencia: Fundación Oficina Valenciana para la SI.

--- (2008): Infobarómetro social de la Comunidad Valenciana 2007. Valencia: Fundación Oficina Valenciana para la SI.

--- (2009): Infobarómetro social de la Comunidad Valenciana 2008. Valencia: Fundación Oficina Valenciana para la SI.

--- (2005): Infobarómetro de e-Gobierno de la Comunidad Valenciana 2005. Valencia: Fundación Oficina Valenciana para la SI.

--- (2007): Infobarómetro de e-Gobierno de la Comunidad Valenciana 2006. Valencia: Fundación Oficina Valenciana para la SI

--- (2009): Infobarómetro de e-Gobierno de la Comunidad Valenciana 2007. Valencia: Fundación Oficina Valenciana para la SI.

--- (2009): Infobarómetro de e-Gobierno de la Comunidad Valenciana 2008. Valencia: Fundación Oficina Valenciana para la SI.

Cities \& Regions World (2005): Declaración política de la 'll Cumbre Mundial de Ciudades y Autoridades Locales sobre la Sociedad de la Información', Il Cumbre Mundial de Ciudades y Autoridades Locales sobre la Sociedad de la Información. Bilbao: Autor. 
EC (2009): Communication from the commission to the european Parliament, the council, the european economic and social Committee and the committee of the regions, europe's digital competitiveness report, volume 2: i2010 - ICT country profiles. COM(2009) 390.

Recuperado el 1 de junio de 2010 de:

http://ec.europa.eu/information society/eeurope/i2010/docs/annual report/2009/sec 20091 104.pdf

Criado Grande, J. I.y Ramilo Araújo, M. C. (2001): "e-Administración, ¿un reto o una nueva moda?. Problemas y perspectivas de futuro en torno a Internet y las tecnologías de la información y comunicación en las Administraciones Públicas del siglo XXI", Revista Vasca de Administración Pública, 61, pp. 1-21.

España. Jefatura de Estado (2007): Ley 11/2007 de Acceso Electrónico de los Ciudadanos a los Servicios Públicos. BOE [Madrid], 150, pp. 27150-27166.

Europa (s.a.): Síntesis de la legislación de la UE. Sociedad de la Información. Recuperado el 5 de septiembre de 2010 de:

http://europa.eu/legislation summaries/information society/index es.htm

Franquet, R.; Ribes, X. ; Soto, M.; Fernández Quijada, D. (2008): "Servicios interactivos en la TDT española: una asignatura pendiente". En Actas y Memoria Final. Congreso Internacional Fundacional AEIC. Santiago de Compostela: Asociación Española de Investigación de la Comunicación.

--- (2009): "La información en la TDT interactiva: una oferta incipiente para unas prácticas periodísticas en transformación”, Trípodos: Revista digital de comunicació, 24, pp 15-29.

Franquet, R., Ribes, X.; Fernández Quijada, D. y Soto, M.T. (2009): "Servicio público e interactividad en la TDT: la política del laissez faire, laissez passer", Sphera Pública, 9, pp 191- 205.

Generalitat Valenciana (2004): Avantic 2004-2010. Comunidad Valenciana. Avanzando con las tecnologías. Valencia: Consellería d'Infraestructures i Transport.

INE (s.a.): "Contabilidad regional de España”. Recuperado el 30 de septiembre de 2010 de: http://www.ine.es/jaxi/menu.do?type=pcaxis\&path=\%2Ft35\%2Fp010\&file=inebase \&L=0

--- (s.a.): "Encuesta sobre equipamiento y uso de nuevas tecnologías de la información y comunicación en los hogares". Recuperado el 30 de septiembre de 2010 de:

http://www.ine.es/jaxi/menu.do?type=pcaxis \&path=\%2Ft25\%2Fp450\&file=inebase \&L=0

--- (s.a.): "Encuesta Anual de Servicios". Recuperado el 30 de septiembre de 2010 de: http://www.ine.es/jaxi/menu.do?type=pcaxis\&path=/t37/e01\&file=inebase

Iturrioz Fanjul, L. (2006): "La administración electrónica en el marco de la sociedad de la información”, Ábaco, 48, pp. 64-68.

Kotsiopoulos, I.; Paparoidamis, N.; Kolomvos, G. y Rentzepopoulos, P. (2009): "Bringing Together and Accelerating eGovernment Research in the EU. eGovernment evolution towards 2020 Report”. Brussels: DG Information Society and Media. European Commission. 
Recuperado el 3 de febrero de 2010 de:

http://ec.europa.eu/information society/activities/egovernment/studies/trendswatch/reports/eg ov evolution towards 2020.pdf

Marzal, J. J. y Casero, A. (2009): "Las políticas de comunicación ante la implantación de la TDT en España: balance crítico y retos pendientes", Sphera Publica, 9, pp. 95-113.

Ministerio de Industria, Comercio y Turismo (s.a.): "Plan Avanza 2". Recuperado el 5 de enero de 2010 de:

http://www.planavanza.es/InformacionGeneral/ResumenEjecutivo2/Documents/PlanAvanza2. $\underline{\text { pdf }}$

Ministerio de Industria, Comercio y Turismo (s.a.), "Plan Avanza". Recuperado el 5 de enero de 2010 de:

http://www.planavanza.es/InformacionGeneral/PlanAvanza1/Documents/2a392d4f65d9404fb 83fc3d2f64eceadplan avanza documento completo.pdf

--- (2007): "Plan nacional de Transición a la Televisión Digital Terrestre". Recuperado el 5 de enero de 2010 de: http://www.impulsatdt.es/pdf/plan-nacional-transicion-tdt.pdf.

--- (2010): Real Decreto 365/2010 por el que se regula la asignación de los múltiples de la TDT tras el cese de las emisiones de televisión terrestre con tecnología analógica. BOE [Madrid], 81, pp. 30750-30762.

Ministerio de la Presidencia (s.a.): "Observatorio de la Administración electrónica. Estudios sobre la situación de la Administración electrónica” Recuperado el 20 de abril de 2010 de: http://www.obsae.map.es/jahia/Jahia/pid/144

Murciano Martínez, M. (2009): "Sociedad de la Información y desarrollo desigual en los espacios comunicativos locales: una aproximación cuantitativa”, en García Hernández, C. y Gómez García, R. (eds.), Comunicación e interdisciplina. Monterrey-México: Universidad Autónoma de Nuevo León-AMIC, pp. 23-52.

ONU-UIT (2004a): Plan de Acción, Cumbre Mundial sobre la Sociedad de la Información, Ginebra, 2003- Túnez 2005, 12 de mayo, Documento WSIS-03/GENEVA/5-S. Ginebra: Autor.

--- (2004b), Declaración de principios. Construir la sociedad de la información: un desafío global para el nuevo milenio, Cumbre Mundial sobre la Sociedad de la Información, Ginebra, 2003- Túnez 2005, 12 de mayo, Documento WSIS-03/GENEVA/4-S. Ginebra: Autor.

Parlamento Europeo (s.a.): "Consejo europeo de Lisboa

23 y 24 de marzo 2000. Conclusiones de la presidencia". Recuperado el 3 de febrero de 2010 de: http://www.europarl.europa.eu/summits/lis1 es.htm.

Prado, E. y Fernández, D. (2006): "The Role of Public Service Broadcasters in the Era of Convergence. A Case Study of Televisió de Catalunya", Communications \&Strategies, 62, pp 49-69. Recuperado el 20 de abril de 2010 de: http://www.idate.fr/fic/revue telech/148/CS62 PRADO FERNANDEZ.pdf. 
Prado, E.; Franquet, R.; Soto, M.T.; Ribes, X.; Fernández Quijada, D. (2008): "Tipología funcional de la televisión interactiva y de las aplicaciones de interacción con el televisor", Zer, 25, pp. 11-35.

Ramiro Sánchez, A. (2002): Demandas de calidad de la Administración Pública: un derecho de la ciudadanía. Madrid: Dykinson.

Rodríguez, J. R. (2006): "El modelo de Barcelona de administración electrónica: adopción e institucionalización”, UOC papers. Revista sobre la Sociedad del Conocimiento, 3, pp. 1-18.

Tintó i Gimbernat, M. (2002): L’Administració pública en la Societat de la Informació: El règim jurídic de la administració pública electrònica. Tesis doctoral. Barcelona: Universitat Pompeu Fabra.

Zallo, R. (2010): "La política de Comunicación Audiovisual del gobierno socialista (20042009): un giro neoliberal", Revista Latina de Comunicación Social, 65, pp. 14-29. Recuperado el 20 de abril de 2010 de: http://www.revistalatinacs.org/10/art/880 UPV/02 Zallo.html.

\section{Forma de citar este artículo en bibliografías}

Galvez, N.; Martinez, M. (2010): "Políticas SI para la participación ciudadana: análisis regional de la TDT y de la e-Administración”, en Revista PANGEA, 1, páginas 177 a 198. Red Académica Iberoamericana de Comunicación. Recuperado el __ de ___ de 2 de: http://revistaraic.files.wordpress.com/2010/12/01-01-109.pdf

\section{Financiación:}

Proyecto subvencionado por el Ministerio de Educación y Ciencia, Plan I+D+i, CSO200800587/SOCI (director: Marcial Murciano)

\section{Agradecimientos:}

A Mar Iglesias García, por su trabajo en la revisión, presentación y comunicación del estudio en el Congreso Internacional Latina de Comunicación Social.

\section{Breve CV}

Natalia Papí Gálvez (Alicante, 1975)

Departamento de Comunicación y Psicología Social

Facultad de Ciencias Económicas y Empresariales

Universidad de Alicante, UA 
Campus San Vicente del Raspeig, Ap. 99. 03080, Alicante, Edificio de Ciencias Sociales Teléfono 34965909641

Natalia.p@ua.es

Licenciada en Sociología (1999), especialidad en Marketing y Publicidad, con premio extraordinario por la UA y Doctora (2004), premio extraordinario de doctorado. Desde 2001, docente en la licenciatura de Publicidad y Relaciones Públicas de la UA en "Introducción a la investigación de medios publicitarios" y, posteriormente, en doctorado. Obtuvo una beca de investigación por la Dirección General de la Dona (1999). A partir de 2001 disfrutó de la beca FPI nacional en la Facultad de Ciencias de la Información de la Universidad Complutense Madrid. Ha colaborado y dirigido varias investigaciones I+D relacionadas con la comunicación, las nuevas tecnologías y los estudios de género. Tiene más de una docena de publicaciones centradas en estas líneas de trabajo. Algunas listadas en http://www.ua.es/dpto/dcps/personal/npapig.html.

Marcial Murciano Martínez (Barcelona, 1948)

Dpto. de Periodismo.

Universidad Autónoma de Barcelona, UAB.

08193 Bellaterra Barcelona España.

Tel. 935811796.

Marcial.murciano@uab.cat

Catedrático en la Facultad de Ciencias de la comunicación de la Universidad Autónoma de Barcelona. Licenciado en Periodismo y Doctor en Ciencias de la Información, por la UAB. Profesor de la Facultad de Ciencias de la Comunicación de la Universidad Autónoma de Barcelona, desde 1977. Director de varios proyectos I+D relacionados con las políticas de comunicación y nuevas tecnologías. Destaca: "Nueva tecnología y cambio comunicativo en las comunidades autónomas Españolas" y "Las políticas publicas de impulso a la sociedad de la información en España: evaluación de las estrategias y actuaciones en nueve comunidades autónomas". Entre sus líneas de investigación, cabe destacar la comunicación internacional, el profesional de la comunicación, las políticas de comunicación y la sociedad de la información. Tiene más de cinco libros: La comunicación internacional; Países y Medios de Comunicación; Aspectos de la comunicación internacional; Estructura y dinámica de la comunicación internacional; La profesión de periodista; Políticas de comunicación o La prensa y la cooperación internacional. Algunos de sus artículos pueden ser consultados en: http://dialnet.unirioja.es/servlet/busquedadoc?db=1\&t=marcial+murciano\&td=todo \&x=0\&y=0 